\title{
Adaptive Optics for Visual Simulation
}

\author{
Enrique Josua Fernández \\ Laboratorio de Óptica, Instituto Universitario de investigación en Óptica y Nanofísica (IUiOyN), Universidad de Murcia, \\ Campus de Espinardo (Edificio 34), 30100 Murcia, Spain
}

Correspondence should be addressed to Enrique Josua Fernández, enriquej@um.es

Received 13 November 2012; Accepted 29 November 2012

Academic Editors: Y. S. Kivshar, V. R. Soma, Y. Tsuji, and S. F. Yu

Copyright ( 2012 Enrique Josua Fernández. This is an open access article distributed under the Creative Commons Attribution License, which permits unrestricted use, distribution, and reproduction in any medium, provided the original work is properly cited.

\begin{abstract}
A revision of the current state-of-the-art adaptive optics technology for visual sciences is provided. The human eye, as an optical system able to generate images onto the retina, exhibits optical aberrations. Those are continuously changing with time, and they are different for every subject. Adaptive optics is the technology permitting the manipulation of the aberrations, and eventually their correction. Across the different applications of adaptive optics, the current paper focuses on visual simulation. These systems are capable of manipulating the ocular aberrations and simultaneous visual testing though the modified aberrations on real eyes. Some applications of the visual simulators presented in this work are the study of the neural adaptation to the aberrations, the influence of aberrations on accommodation, and the recent development of binocular adaptive optics visual simulators allowing the study of stereopsis.
\end{abstract}

\section{The Eye and Its Aberrations}

Vision is a fascinating puzzle involving different aspects. In a first stage, light emitted or reflected by external objects reaches the eye. The image of the object is projected onto the observer's retina by the optics of the eye. The information contained in the image is to be processed and transmitted from the retina to the brain by neural cells. The brain is finally in charge of the psychological interpretation of the scene for a useful perception of our surrounding reality. Each part of the complete process of vision is complex enough for requiring a separate analysis. The first part of vision pertains solely the optical aspects of formation of the images, and it is usually termed the optical or first stage. In this work, several aspects related to the optical stage will be shown, in particular, the connection of vision and the optical quality of the eye, and how adaptive optics has dramatically changed our methods and approaches for the study and understanding of vision.

It seems appropriate starting with a description from an optical point of view of the eye. The eye is a complex system showing a tremendous histological richness. However, when studying the formation of images on the retina from the real world only some parts of the eye are relevant [1]. In the following, some fundamental parts of the eye are revisited. Some of them are shown in Figure 1.

The first element that light encounters coming from the object or scene is the cornea. The latter provides approximately two-thirds of the total optical power of the eye. Considering solely the optical properties, the cornea might be characterized by the radii of curvature of its anterior and posterior surface, thickness, and refractive index, which exhibits an average value of 1.38 in the normal eye. After the cornea, the light finds the anterior chamber. This is a space filled with the aqueous humor, essentially compounded by water, showing a thickness of $3.05 \mathrm{~mm}$ and a refractive index of 1.34. The next relevant element for the light in its travel to the retina is the iris. This is a circular muscle which defines the eye pupil, where the light beam is limited through. The iris diameter can change because of a number of factors. Across others, the most relevant changes occur in response to illumination variations in the scene and changes in the distance to the fixation point. After the iris, the posterior chamber is found. This is defined as the space filled with aqueous humor and limited by the crystalline lens, which acts as its posterior limit. The crystalline lens 
is beyond doubt one of the most fascinating and complex optical elements of the eye. It provides to the eye, together with the cornea, the rest of the necessary optical power for correctly focusing images on the retina. It is compounded by a large number of concentric layers, of different thickness and cellular age. This particular structure grants the lens with a complex distribution of refractive index along its optical axis, sometimes compared with a GRIN, or gradient index, lens. Its external shape resembles a biconvex lens, slightly more curved at the posterior outer surface, showing a total average thickness of $4 \mathrm{~mm}$. For the study of the eye from an optical point of view, a first useful approximation for modeling the lens is taking a single effective refractive index of 1.42. The crystalline lens is enclosed by a thin membrane endowed with elastic properties, mostly compounded by type IV collagen, known as the capsule. The stiffness of the capsule is larger in the young eye, determining the external shape of the crystalline lens. The capsule is suspended equatorially by means of a delicate network of elastic fibers known as the zonula. The most external parts of the fibers are inserted into the ciliary muscle by the ciliary processes. The ciliary muscle is in direct contact with the sclerotica, which is the most outer part of the eye. As it will be shown later in this work with more details, all these elements described previously are interconnected and they are of relevance for the understanding of the accommodation, a fundamental capability of the eye.

The vitreous humor is located right after the crystalline lens. It shows a gel-like texture with a refractive index similar to that of the aqueous humor. Its role is mostly keeping the rest of elements fixed at the right position in the eye and protecting the retina. The retina is formed by a multitude of cells of different kinds, exhibiting a highly organized structure of different layers grouping similar cells. It is responsible for the phototransduction of the optical image into chemical and physical signals which are sent and impulses which are sent to the brain by means of specialized neural cells. In the brain these signals are decoded by a delicate mesh of neurons for the final subjective psychological perception of vision. The zone of the retina where the images are projected for accurate vision is called the fovea. The latter corresponds with the retinal area most populated of photoreceptors. The photoreceptors are the cells responsible for the detection of light.

In the study of the optical aspects and formation of images in the eye, the use of some axes is convenient. The optical axis of the eye is defined as the imaginary line containing the centers of curvature of the different surfaces of the elements compounding the eye. In the human eyes the optical axis does not intersect the fovea $[2,3]$. That is the main reason for introducing the visual axis or line of sight. This axis is defined as the imaginary line connecting the fixation point, where the eye is looking at, and the fovea. The angle between the two axes is commonly named as alpha angle, although some alternative definitions can be found in the literature.

In the previous description, all elements of the eye are modeled by ideal surfaces of given radii of curvature and known refractive indexes. This kind of modeling is extremely

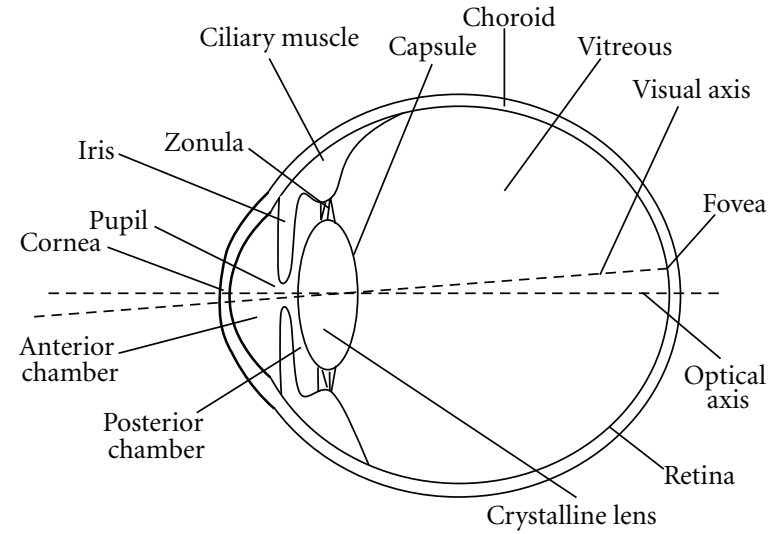

Figure 1

useful for the understanding of several aspects of image formation. This description of the eye can provide a simple explanation of the generation of blurred retinal images in the presence of refractive errors, approximate image size, and so forth. From this model one could infer that optical images generated on the retina are somehow comparable to those produced by a regular optical instrument, in terms of quality. The reality is nevertheless more complicated [4-6]. In the real eye, none of the surface can be generally described by a simple radius of curvature, not even by a conic surface [7]. The surfaces exhibit deviations from ideal surfaces inherent to any biological tissue. The different surfaces separating media of distinct refractive indexes are not aligned neither their hypothetical centers of curvature lay on a common axis. That makes the different surfaces appear as misaligned to each other in the eye. In addition, the optics of the eye does not remain steady, but on the contrary it presents continuous fluctuations in time [8-11]. Those come mainly from the changes in the crystalline lens induced by the physiological tension of the ciliary muscle. The tear film, the intraocular pressure, the changes, and movement associated with the ocular humors are other factors preventing a steady situation in the eye. All these circumstances result in that the retinal images finally formed on the observers' retina were far from perfect images. Retinal images are affected and degraded by ocular aberrations $[4,5,12-18]$. Those are the deviation of the wavefront of the incoming light from a paraxial situation, where all light rays would converge in a single point on the retina. Therefore, the interest in measuring and characterizing the effect of aberrations and their dynamics on vision is fundamental [12]. In the last years, many efforts have been devoted to understand to what extent these aberrations can affect the perception in vision. In addition, correcting those aberrations would permit to achieve unprecedented resolution in the retinal images recorded in the living eye, with promising applications in the diagnosis and treatment of several retinal conditions. In this scenario, adaptive optics (AO) is playing a major role as it will be shown in the following. 


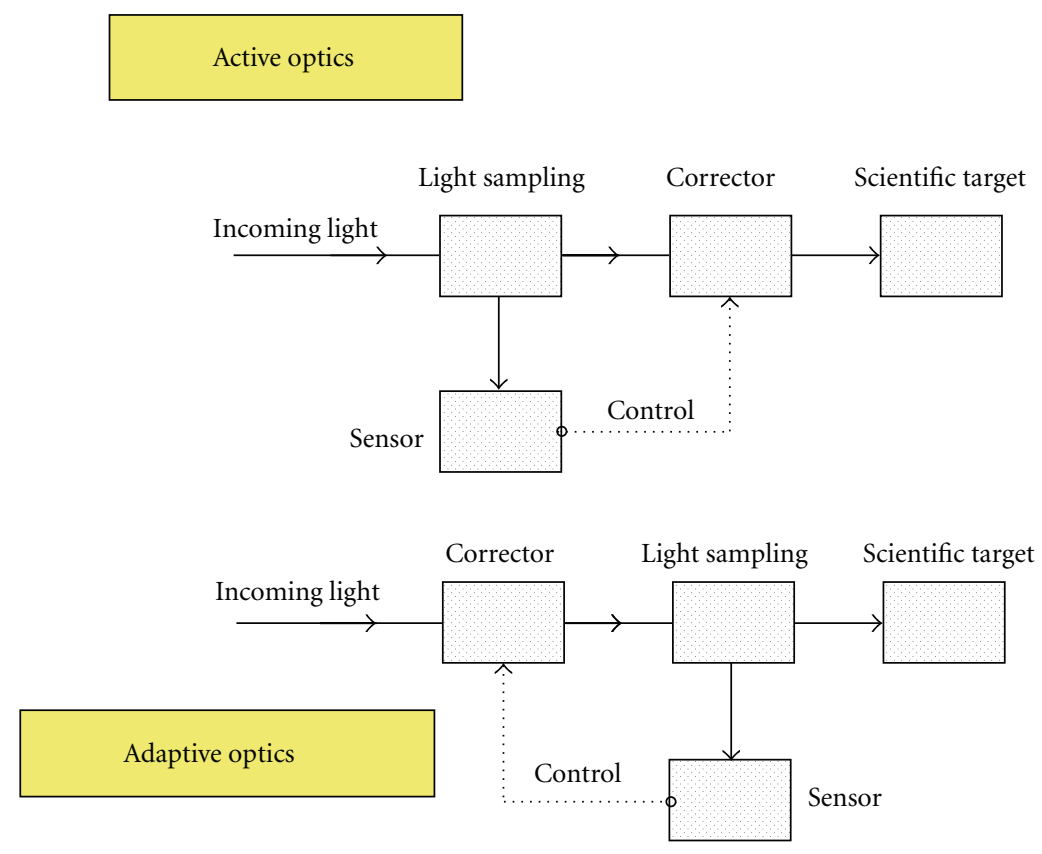

FiguRe 2

\section{Adaptive Optics}

A first simple definition for adaptive optics could be the optical technique allowing the measurement and subsequent correction of optical aberrations. In spite of the simplicity of the previous definition, the two fundamental concepts sustaining $\mathrm{AO}$ are clearly present. A first key point is the measurement of aberrations, that must be performed is a robust manner [8-10], and faster than the typical temporal variation which in intended to be later corrected. The other evident pillar of $\mathrm{AO}$ is the correction of aberrations. The AO system must allow for the controlled manipulation of the wavefront, once it has been estimated. The $\mathrm{AO}$ aberration correction concept inherently includes the measurement of the effect of the correction over the wavefront so that closed loop control of aberration can be eventually performed. There is an alternative concept generally known as active optics which performs the correction after the measurement of the aberrations, with no possibility of closed loop operation. Consequently, in active optics there is no means of measuring the effect of the correction over the wavefront. Figure 2 shows schematically a diagram of active and adaptive optics.

The first proposal of the use of AO technology for civil purposes was suggested in the context of astronomical optics in the year 1953 [19], around thirty years earlier of the experimental demonstration of the technique. Irrespectively of the optical quality and the aperture of the telescopes, light passing through the atmosphere is seriously degraded by effect of the changing temperature, gradients of pressure, winds, and in general any disturbance able to locally alter the refractive index of the medium. Therefore, the images recorded at the ground level are systematical affected and degraded by all these factors. $\mathrm{AO}$ was presented as the technology providing the solution to the atmospheric turbulence problem over the astronomical images recorded in the ground-based telescopes. Nowadays, every professional telescope in the world is endowed with $\mathrm{AO}$ for the atmospheric aberration correction. The field of astronomical optics was the only one benefiting from $\mathrm{AO}$ during a couple of decades, approximately from the eighties to nineties. Probably the elevated cost of the AO systems prevented other fields from exploring the possibilities of AO. It could even be said with some humor that it was especially true in the many other fields unfortunately not enjoying of astronomical funds. In the context of vision sciences, it was not until 1989 [20] when some of the concepts from $\mathrm{AO}$ were somehow applied in the eye. In that work, the use of a deformable mirror for the static compensation of the ocular aberrations was reported. The final target of the correction was the improvement of the resolution achieved on the retinal images obtained with a scanning laser ophthalmoscope. The correction of aberrations was restricted to the existing astigmatism.

The progress and evolution of $\mathrm{AO}$ in the eye was firstly sustained on the development of reliable methods for measuring the ocular aberrations. Among the many different techniques for subjectively estimating the ocular aberrations, the Hartmann-Shack $(\mathrm{H}-\mathrm{S})$ wavefront sensor has been the most widely employed method in the context of vision. That technique was imported from astronomical optics as well. The first proposal of such technique for the study of the eye was reported in 1991 [21]. In that work, the H-S wavefront sensor was proposed for estimating the topography of the living cornea. The experimental demonstration of such technique in the eye, measuring the total aberrations, was definitively published in 1994 [22]. Other works have contributed for notably improving the efficiency of the algorithms [23], improving the technique to the level that 
it has achieved today. IR range is the preferred illumination for measuring the aberrations in the eye [24-27]. The use of the H-S wavefront sensor allowed for measuring highorder aberrations, consequently opening the door to their subsequent correction [24, 28]. It was in 1994 when the first demonstration of high-order static aberration correction was published [29]. Some years later, in 2001, the first closedloop aberration corrections in real time performed in the living eye were finally reported $[11,30]$. In the work of Liang, static ocular aberration correction was accomplished by means of a deformable mirror, for the first time beyond defocus and astigmatism. Two applications of $\mathrm{AO}$ in the eye were surveyed: vision through a virtually perfect optics and retinal imaging of high resolution. These uses of the AO have been widely explored by the scientific community in the last years. Regarding the field of retinal imaging, a number of important results arouse from the application of $\mathrm{AO}$ to the different imaging techniques. Almost every technique has been merged with $\mathrm{AO}$ in the last decade, providing new insights into the existing knowledge of the retinal structure in vivo. Flood illumination fundus cameras were the first benefiting from the utilization of $\mathrm{AO}$, permitting the in vivo recording of the photoreceptors mosaic, even allowing the classification of the three types of them [31]. AO combined with modern laser scanning ophthalmoscopes is providing a unique tool for studying the intimate morphology of the living retina [32], also unveiling interesting aspects of vision. Last technique benefiting from $\mathrm{AO}$ was optical coherence tomography (OCT) $[26,33-35]$. OCT combines high axial resolution and fast scanning rates. $\mathrm{AO}$ has allowed to study the true three-dimensional architecture of the living retina with important applications in the understanding of many retinal conditions $[25,27,36-40]$.

\section{Adaptive Optics and Visual Simulation}

The use of $\mathrm{AO}$ for providing a virtually perfect vision was the other evident application of the technique [29]. Correcting the ocular aberrations should allow vision for reaching its physiological and perceptual limits. The experiments showed an increase in the visual performance in absence of aberrations. Nevertheless, correcting high-order aberrations in the normal young eye produces a limited impact in vision. Some works have studied the distribution of aberrations beyond defocus and astigmatism $[14,18]$. It is in the pathologic eye, for instance, those affected by corneal conditions as keratoconus, where $\mathrm{AO}$ correction of high order aberrations can really produce a significant benefit in vision. Now it is accepted that high-order aberrations are of a modest importance in the total picture when normal young eyes are considered. In addition, an $\mathrm{AO}$ system uses of a number of elements as the corrector, the wavefront sensor, and so forth which prevent with the current state-of-the-art a system compact enough to be coupled directly to the patient's eye.

AO can operate not only for correcting aberrations but in general for manipulating the wavefront. Aberrations can be partially corrected, selecting which particular terms are modified, and which are left as they appear in the eye for instance. This possibility opens a rich number of experiments

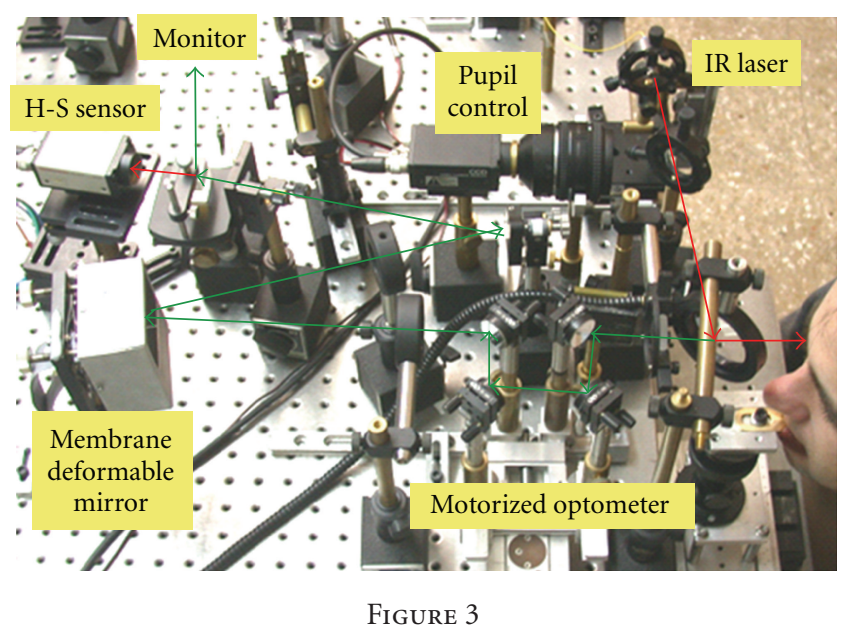

for the better understanding of the relationship among optical quality and vision. It was in 2002 when this paradigm was experimentally demonstrated in the eye for the study of vision $[41,42]$. In the work of Fernández et al., [41], the $\mathrm{AO}$ system was coupled to an additional optical relay for the display of stimuli. Visual testing was enabled simultaneously for the operation of the AO system. The apparatus was presented as a visual simulator. Different wavefronts could be generated and vision through the modified optics tested in parallel.

Figure 3 presents a picture of the first visual simulator system. The complete setup was mounted over an optical table of $1 \mathrm{~m}^{2}$. A membrane deformable mirror with 37 independent actuators acted as the correcting device (OKOFlexible Optical, The Netherlands). Due to the limited amplitude of deformation of the mirror, large amounts of aberrations could not be programmed [43]. In practice, for normal eyes most of the weight of the aberrations is distributed across defocus and astigmatism. Defocus was controlled by a motorized Thorner optometer of high precision, and astigmatism was eventually compensated by introducing trial lens if required. Once static correction of these large aberrations was further investigated, the deformable mirror was able for the real-time compensation of their temporal fluctuations. An external monitor was coupled to the system for the presentation of visual stimuli.

In the first experiment of visual simulation with adaptive optics, a subject with a remarkable coma aberration, in addition of defocus, was asked to complete visual acuity tests while some aberrations were manipulated. Three different situations were explored for understanding the role of aberrations in vision. The coma aberration presented a given axis, causing a marked directionality in the subject's point spread function. In the eye, the existence of such aberration caused an asymmetric blur in the retinal images which should degrade visual performance accordingly. Some amounts of controlled coma aberration were added to the natural aberrations of the subject. From the objective retrieval of the ocular aberration, other optical parameters such as point spread function (PSF) or the modulation transfer function (MTF) could be inferred. Visual acuity 


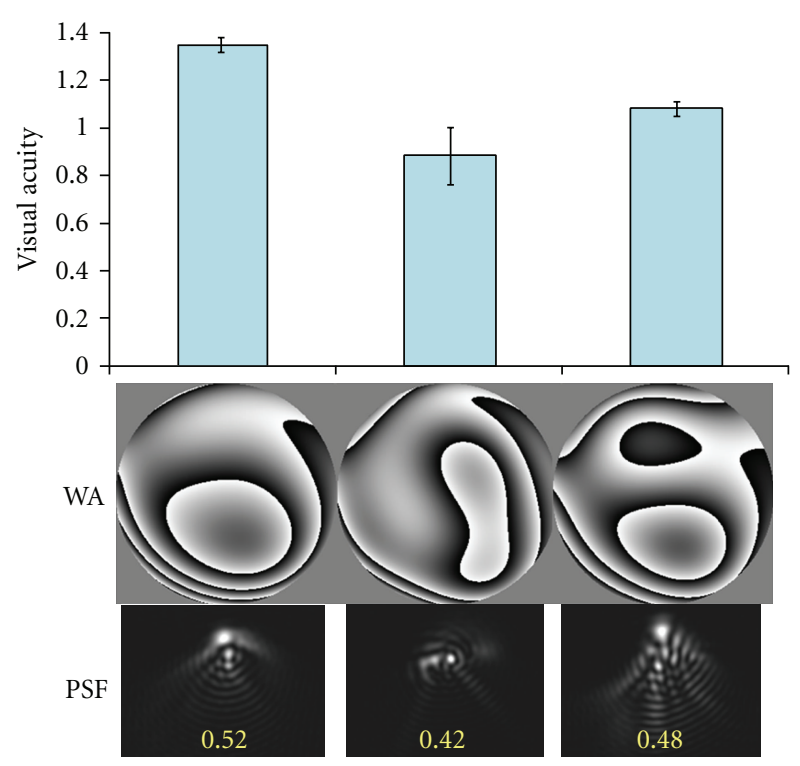

FIGURE 4

was obtained under three cases in the study: vision with natural aberrations; coma aberration added in the same axis than the existing in the subject's eye; the case of $90 \mathrm{deg}$ rotation of the coma aberration, keeping approximately the same modulus than in natural case. The analysis of the MTF revealed that from a purely optical perspective, the quality of the natural and the rotated case were similar; so acuity test obtained from the average of different orientations should not exhibit substantial differences. In the situation of addition of coma aberration, the area of the MTF presented a significant decrease at every frequency, so the poorest acuity was expected in this case. The results were somehow surprising, since adding the same amount of coma aberration to the eye at distinct directions, once corrected of low-order aberrations, caused significant differences in the visual perception of acuity tests. Adding coma aberration in the same direction that the one already present in the eye degraded vision less than the same value at any other direction. This result suggested that vision is coupled to the optical quality of the eye in an unexpected manner. The larger tolerance of vision when retinal images are degraded in a particular direction might be due to a kind of adaptation of the visual system to its own aberrations. Figure 4 shows graphically these findings. The average visual acuity with the standard deviation from the measurements is plotted with the corresponding generated aberrations. Their associated PSFs and the measured RMS of the wavefront are also displayed in Figure 4.

This hypothesis of adaptation to the aberrations was investigated in a different experiment, where the vision of a larger number of subjects was tested with manipulated wavefronts [44]. In such work, the perception of blur was evaluated by using a random pattern showing irregular black spots on a bright background. Monochromatic illumination at $543 \mathrm{~nm}$ was used in order to avoid the possible effects of chromatic aberrations. The ocular aberrations of each subject were recorded. Using the retrieved wavefront, an algorithm generated the appropriate shape for the deformable mirror so that the combination of ocular and mirror wavefronts produced a rotated version of the original ocular aberration. The procedure was performed in closed loop, at real time. This operation guaranteed that the subject was continuously viewing the test through the modified aberration pattern. It must be stressed out that the experiment forced the optical quality of the retinal images projected on the observer to be exactly the same, but for orientation. A two-alternatives forced choice test was programmed. Images through the natural aberrations of the eye were displayed together with the same through the modified version of the aberrations. Subjects were asked for changing the level of perceived blur in the manipulated version of the images until equalization of blur with natural viewing was achieved. For accomplishing the task, the subjects multiplied the rotated version of the wavefront by a factor which globally reduced the level of aberrations, simultaneously in all terms. The results were a systematic perception of larger blur when viewing through a rotated version of the own subjects' aberrations. Since the optical quality of the retinal images was similar in all cases, the different perception of blur had to be produced by neural factors. The proposed explanation to this novel effect was a new kind of adaptation to the own individual aberration pattern. Essentially, the brain could be used to extract information from images degraded in a constant manner, imposed by the subject's ocular aberrations. Any change away from this natural shape degrades perception, even if the optical quality levels are similar. The experiment was interesting since it opened the door to a novel perspective for understanding vision. Many other experiments using AO since then have explored a number of open questions related to adaptation of vision to the ocular aberrations [45-50].

The effects of neural adaptation might become more evident in those eyes suffering of larger monochromatic aberrations. A typical example of highly aberrated optics can be found in those patients with keratoconus. This is a relatively well-studied condition producing a severe deformation of the anterior and posterior surfaces of the cornea. As it has been mentioned before, the effects on the retinal images are usually very strong, degrading the vision of the patients up to dramatic limits. Some other works have used adaptive optics in the context of keratoconic eyes, trying to better understand the capability of the eye for adapting to these large aberrations [51-53].

An intriguing question partially arousing from these experiments of adaptation is the possible effect of the aberrations on accommodation. Aberrations change when the eye accommodates, beyond defocus and astigmatism. Therefore, correcting or altering the aberrations with $\mathrm{AO}$ for far vision could eventually degrade somehow accommodation or near vision, since the aberrations pattern in the eye might be significantly distinct from the natural shape. Many questions are to be answered. These particular points will be treated in the following. 


\section{Adaptive Optics and Accommodation}

Accommodation is possibly one of the most studied functions of the visual system. This feature allows the eye to increase its optical power so that near objects can be correctly focused onto the observer's retina. The augment in power is exclusively caused by the crystalline lens. The intimate mechanism of accommodation involves several components. A simplified picture of the accommodation is presented in the following. The blurred retinal image produced by a near object originated, through a number of neural channels, the contraction of the ciliary muscle. The contraction of the muscle reduces the space around the crystalline lens in the equatorial plane. The first consequence is the reduction of the tension in the zonula. The capsule containing the crystalline lens and the lens itself are then able to compress towards their relaxed state. The anterior and posterior surfaces of the crystalline lens experience an increase in their curvature. The total thickness of the lens also enlarges. All these geometrical changes in the crystalline lens produce the global effect of increasing its power. Several aspects concerning the mechanism of accommodation are still unveiled. An intriguing question is how the visual system is able to change the power of the eye in the correct direction. In a situation where the eye is focusing a target situated at middle distance, a change of the stimulus or a variation on its position will immediately produce a blurred image onto the retina [54]. Defocus alone does not provide any cue about the direction of change, since the degradation of the image is symmetrical around the initial image plane [55]. The visual system is, however, known to produce the accommodation in the right direction in most of the situations [56]. In this context, the existing chromatic aberration of the eye has been proposed and demonstrated as a source of asymmetry in the retinal images providing, at least in part, information about the factual direction for accommodation [57-60]. Other optical cues have been proposed, for instance, the microfluctuations in accommodation [61]. More recently the ocular aberrations have been also proposed as a possible source of asymmetry in the defocused retinal images which eventually could provide an additional cue for determining the direction of accommodation [62]. Adaptive optics was applied for studying accommodation, and the possible role of monochromatic aberrations in the visual response to defocused stimuli [34]. The experiment consisted in the correction of monochromatic aberrations during accommodation induced by an abrupt change of $1.5 \mathrm{D}$ in the vergence of the stimulus. The variation in the perceived position of the stimulus was generated by using a motorized Badal optometer. Two subjects with normal accommodation capability and normal values for their ocular aberrations participated in the experiment. The procedure was performed under monocular vision and with monochromatic illumination. These two factors are related with known optical cues for accommodation. The experiment isolated all other optical cues but high-order monochromatic aberrations. Defocus was uncorrected for allowing the subjects to freely change its value. Several parameters related to the accommodation response were obtained with and without adaptive optics aberration correction. The final level of achieved accommodation was measured under the two conditions. No significant variation of this particular parameter was detected, meaning that the precision of the accommodation was independent of the existing ocular aberrations. This was a somehow surprising result, since it showed that the quality of the retinal images, when they are distorted by normal levels of monochromatic aberrations, is not a key factor in the eye for finding the best focused retinal image. Another parameter of the accommodation studied in the experiment was the latency time of the visual response. The latency can be understood as the time gap between the blurring of the stimulus and the instant when the eye begins the accommodation. This latency was also found to remain independent of the correction of monochromatic aberrations. The other temporal factor involved in the dynamic of the accommodation and retrieved in the experiment was the response time. This parameter was defined as the time that the accommodation is changing from the initial value to the final level achieved. For this variable, a significant increment in its absolute value when correcting the monochromatic aberrations was measured. It indicated that the compensation of the aberrations degraded this particular parameter, causing an increment in the required time to achieve the final level of accommodation for focusing the stimulus. That was an unexpected result, since it showed for the first time an important function of the visual system as the accommodation suffering a deterioration directly produced by the correction of the monochromatic aberrations. A possible conclusion of that work is that natural aberrations play a role in some visual functions, as the accommodation, and their correction might not be reasonable in all cases. Others works have studied the possible relationship between accommodation and monochromatic aberrations. Some experiments have reported the lack of effect of correcting aberrations in some subjects [63-65], while other have shown a clear benefit in the dynamic of the accommodation associated to the aberrations compensation [66].

Possibly for some subjects monochromatic aberrations are a useful cue in accommodation, and consequently they employ the asymmetry in the retinal images for enhancing the dynamics. It is reasonable then that those subject experience a degradation in their accommodation when aberrations are compensated. On the other hand, other subjects might not use such information, and consequently the correction of retinal images through the aberration compensation enhances some of their accommodation parameters because of a better retinal image. Depending on the level of aberrations, it might be also reasonable that for some subjects the correction of monochromatic aberrations was irrelevant for the accommodation function. In any case, the question remains unsolved and possible future experiments will provide additional information about the connection between accommodation and monochromatic aberration. 


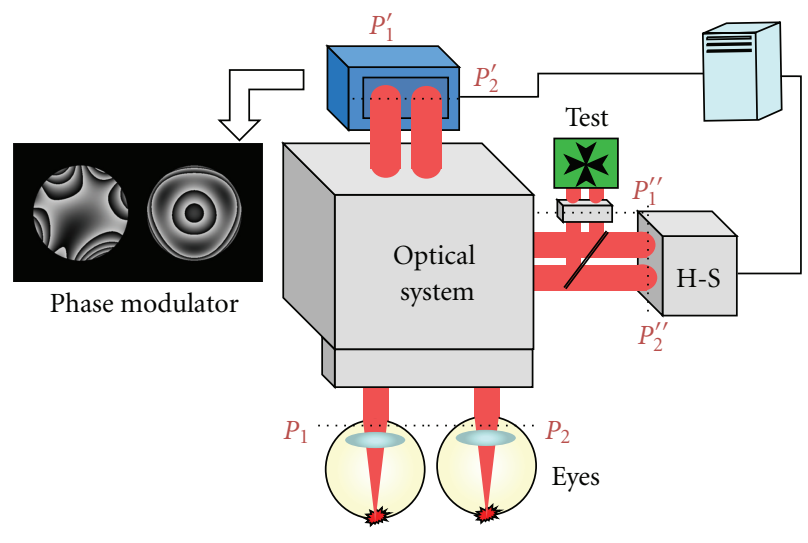

Figure 5

\section{Binocular AO}

Normal vision is not monocular vision. At the beginnings of the development of $\mathrm{AO}$ in the eye, all the experiments were devised under monocular vision. Binocular vision provides a number of advantages for our perception that cannot be neglected [67], for instance, the capability for perceiving depth in the natural scenes. Because of the necessity for objectively measuring binocular refraction, some optometers capable of simultaneous defocus estimation of the two pupils were developed in the recent past [68-71]. They were the antecedent systems to the first binocular wavefront sensors. In 2008, Kobayashi et al. reported a system endowed with two Hartmann-Shack wavefront sensors specifically designed for binocular estimation of the aberrations from the two eyes [72]. The system operated in open view. With this apparatus, the subjects could undergo visual testing while the measurement of their ocular aberrations was taking place. This first approach of replicating the sensor brought about the duplication of the cost of the system, together with an increase in the complexity of the control of the setup. In the same year, another alternative to this first solution for the binocular objective estimation of the ocular aberrations was presented in the work of Hampson et al. [73]. In this other approach, a design allowing the estimation of the aberrations from both eyes employing a single sensor was reported. Using a Hartmann-Shack-based apparatus, the light emerging from each pupil was redirected into the system, keeping along the optical relays the two beams spatially resolved. The two eyes exit pupils were projected on the surface of the wavefront sensor, so that the camera could obtain in a single frame the spots from both pupils. Appropriate postprocessing allowed the retrieval of the ocular aberrations from both eyes. The advantages of such apparatus were evident in terms of cost and complexity.

It was not until 2009 that the first binocular adaptive optics system was reported and successfully applied on real eyes [74]. The most remarkable feature of this system, specifically designed for operating under binocular vision, was the employment of a single correcting device in combination with a single wavefront sensor. The latter operated a similar approach of that reported by Hampson [73], separating the two pupils on the surface of the detector. The correcting device $[74,75]$ followed an analogous principle for managing the two pupils. Those were simultaneously projected on its surface, still spatially resolved, and independently driven from the computer. The fundamental issue arising from this configuration was procuring enough resolution on the corrector for manipulating aberrations, including high-order aberrations, from both pupils with sufficient accuracy. In order to solve this obstacle, a liquid crystalbased correcting device was incorporated in the system. This kind of correctors was a basic pillar for the understanding of the current state-of-the-art of binocular adaptive optics visual simulation. Figure 5 shows a diagram of a binocular AO visual simulator. Their most important and attractive characteristic was possibly the enormous resolution that they exhibited, particularly as compared with deformable mirrors. In the first binocular visual simulator, a commercial spatial light modulator (LCOS-SLM X10468-04, Hamamatsu Photonics, Japan) with SVGA resolution was incorporated. The total number of pixels was $800 \times 600$, selecting approximately 58000 pixels for each pupil. The device allowed keeping a reasonable number of pixels dedicated for the control of the aberrations from each eye. The experimental system incorporated an optical relay for displaying binocular stimuli. The capability of the apparatus was demonstrated by testing the impact of different combinations of spherical aberration on vision. For such purpose, the contrast sensitivity of a subject at $7.8 \mathrm{c} / \mathrm{deg}$ in green light was obtained for different conditions. The contrast sensitivity was obtained by using a two-alternative forced choice test, showing a panel with the target grating and different contrast and other with homogenous background. The subject answered which one was displaying the grating. The time for displaying the stimuli was $500 \mathrm{~ms}$. The grating subtended $1 \mathrm{deg}$, assuring isoplanatic conditions at the retina. The contrast of the grating was set randomly. A psychometric curve was obtained for inferring the value of the contrast sensitivity. That was estimated as the detection threshold at $75 \%$ of confidence. The experiment was repeated under monocular and binocular conditions to discern the influence of binocular summation. The value of spherical aberration was $\pm 0.2 \mu \mathrm{m}$ for a pupil of diameter $4 \mathrm{~mm}$. The experiment accomplished in this work was presented as a demonstration of the potential of the technique. A single subject underwent contrast sensitivity testing. Therefore, extrapolation to general conclusions should be taken with care. Still, some interesting results were obtained. Those are grouped in Figure 6.

The plot shows the contrast sensitivity obtained for different combinations of spherical aberration. The signs + and - indicate the value of the spherical aberration added on the eyes. The position of the value in the brackets represents which eye was affected for each value, being the first and second values for the left and the right eye, respectively. The subject reported his dominant eye to be the left one. Systematically, keeping the dominant eye with the natural aberrations caused a better visual acuity. That was particularly evident when the results were compared with those corresponding to the reversal cases, 
where spherical aberration was added to the nondominant eye. Another interesting result was that the degradation of visual acuity was more dramatic when positive spherical aberration was added to the dominant eye, in absolute values. This particular eye exhibited a larger tolerance to negative spherical aberration.

Among the different advantages that binocular vision provides over our visual perception, stereopsis is one of the most evident features. Stereopsis is intimately connected with the perception of depth [76-79]. The sensation of depth can be also generated from a number of monocular cues. The previous knowledge of the size of an object can produce the estimation of its relative distance regarding other parts of the scene, therefore creating the psychological experience of depth. Other classical monocular cues are the motion parallax, the perspective, texture gradient, accommodation, defocus blur, and so forth. Stereopsis is often defined as the capability for perceiving the depth in a scene originated exclusively for the distinct position of the images on each retina, or more commonly referred to as retinal disparity. This kind of retinal parallax, produced by the relatively shifted perspective of the object each eye has been widely studied in the context of visual perception.

Stereopsis involves a delicate neural processing in addition to the purely optical or geometrical stage. Actually, stereopsis has traditionally been studied in the context of psychology of the perception. Some recent attempts for introducing the possible impact of the ocular optical quality over stereopsis have been reported $[80,81]$. In this direction, the possible influence on stereopsis due to the changes in the ocular aberrations has been considered [82]. Such changes can be found as a consequence of refractive surgery. Adaptive optics provides the ideal tool for characterizing the actual impact of aberrations on stereopsis. Not only low-order aberrations, as defocus and astigmatism can be tested, but in general any required aberration. In the work of Fernandez et al. [83], the stereopsis was evaluated degrading the wavefront with different aberrations by using a binocular adaptive optics visual simulator. The system incorporated two different internal displays for projecting distinct retinal images over each retina. This permitted the generation of retinal disparity, indistinguishable from an optical perspective to that created with a real scene in front of the subject. The stereopsis was tested through the measurement of the stereoacuity. Stereoacuity provides a numerical estimate of the capability of the subject for detecting changes in depth associated exclusively to retinal disparity. Actually, stereoacuity is the minimum retinal disparity, given as the subtended angle, causing the perception of depth. Using separate displays for each eye guaranteed that no other monocular cue was involved in the experiment. Random dot stereograms were used for obtaining stereoacuity. Random dot stereograms are a useful tool for understanding pure stereopsis, particularly global stereopsis. They were introduced by Julesz in the 70s [84] and since then these have been widely employed for binocular vision research. In the aforementioned work, pure defocus was first added under different conditions. Selecting a pupil diameter of $4 \mathrm{~mm}$, stereoacuity was retrieved for natural vision with low-order

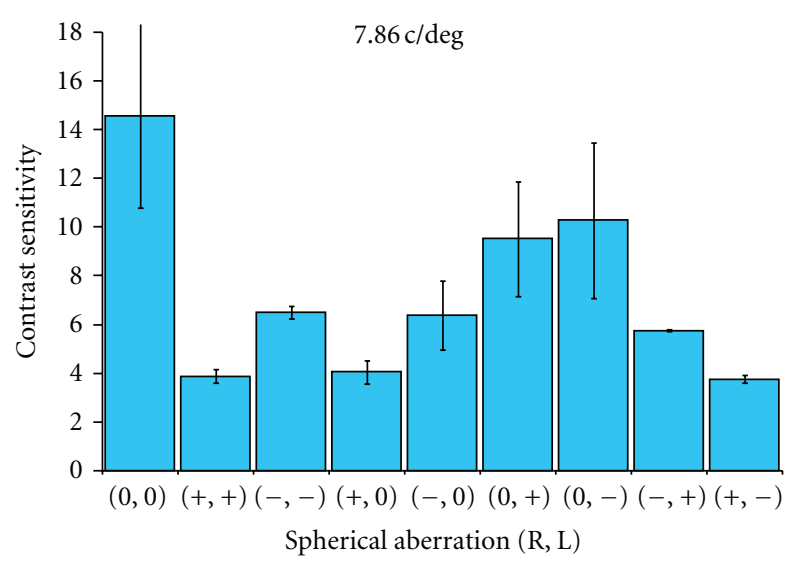

Figure 6

refractive correction (including defocus and astigmatism), addition of $1 \mathrm{D}$ of defocus in both eyes and $1 \mathrm{D}$ of defocus in one of the eyes. The results showed a progressive loss of stereoacuity in the different conditions previously presented, in the same order that they have been mentioned. In particular, for one of the subjects the values of stereoacuity were 4,6 , and $8 \mathrm{sec}$, respectively. The worst case regarding the value of stereoacuity was reported when asymmetric induction of defocus, rather than in the bilateral case. This result confirmed previous studies about the relative impact of blur, caused by defocus or in general low-order aberrations, on stereopsis. There is a large number of works which have considered other factors as the loss of contrast or luminance of the stimuli and their effect on stereopsis. Most of them could be considered from a wide perspective as different outcomes of defocus [85-92]. It is relatively well established that monocular degradation of the retinal images, through defocus, contrast, or luminance, decreases stereopsis more than the corresponding bilateral degradation. The work of Fernández et al. [83] introduced for the first time in the literature the evaluation of the impact on stereopsis of a high-order aberration generated by adaptive optics. The trefoil aberration was selected and applied both binocular and monocular while simultaneous measurement of the stereoacuity was performed. The obtained trend followed that typically occurring for defocus. The addition of trefoil in a single eye produced a larger degradation on stereopsis than the bilateral case. The obtained values of stereoacuity were 4,13 , and $18 \mathrm{sec}$ for natural vision (with no additional aberrations), unilateral and bilateral addition of pure trefoil, respectively. An evolution of this experimental setup was reported by Schwarz [93]. The new apparatus incorporated an additional liquid crystal operating in transmission for the manipulation of the pupils. This other liquid crystal acted as an amplitude modulation device, manipulating pupils' size and their relative position during the measurements, still keeping the capability for phase modulation with the other correcting device. 


\section{Conclusions}

In this paper, different results and applications of adaptive optics have been covered. In particular, special attention has been paid to visual simulation. There are some other related topics which have not been presented. In this context, possibly one of the most promising fields is the employment of adaptive optics for the design of new ophthalmic elements $[94,95]$. This is one of the applications that have benefitted more from adaptive optics visual simulation in the last years. Due to its extension and complexity, this topic has not been tackled in the current paper, but it is definitely an important application which has already changed some paradigms in optical design. The technique permits for the first time the virtual implementation of the designed optics on real eyes before manufacturing. Adaptive optics can reproduce the wavefront, and a visual simulator apparatus can allow patients to see through the optics, eventually testing the expected visual benefit. Moreover, for customized solutions incorporating the compensation of individual ocular aberrations by visual simulators provides not only the platform for measuring these aberrations but the station capable of testing the effects, again prior to the practical piecing of the optics. That new procedure significantly reduces cost and provides a faster method for optimizing the final element to be coupled to the eye. As an example, it has been successfully demonstrated for intraocular lenses, contact lenses and for studying the effect of spherical aberrations for extending the depth of field [96-101].

From a more basic science perspective, another interesting topic where adaptive optics visual simulators and related systems are helping to answer many fundamental questions is the true impact of aberrations on vision. Once ocular aberrations have been correctly measured, the question arising is their influence on vision. Many works have reported progress in this field from the early beginning of the adaptive optics in vision $[29,49,102-120]$.

There are other exciting applications of adaptive optics not covered in the current work. High-resolution retinal imaging is unveiling the intimate structure and function of the retina. Some experiments are connecting the retinal structure and the visual function, answering some basic questions about how we see the world $[48,121-130]$.

Adaptive optics in visual sciences is in constant evolution. In this paper, several success and applications of the technology have been presented, but probably many others are to come in the near future. Other interesting reviews on adaptive optics have been published, where the interested reader will find a distinct focus and very useful information about the technique with additional valuable references $[131,132]$. Adaptive optics has dramatically changed our knowledge about the eye and vision. A natural step would be the incorporation of such modality in the clinical practice, so that patients and clinicians can benefit from that. The state-of-the-art adaptive optics is now mature enough to be incorporated into a rich variety of instruments. The future will probably show a progressive merging of adaptive optics into the most widely used ophthalmoscopes for increasing their resolution up to cellular level. The visual simulators are already in the market for advanced refraction, and they have the potential for becoming the reference phoropters.

\section{References}

[1] Y. Le Grand and S. G. El Hage, Physiological Optics, Springer, Berlin, Germany, 1980.

[2] J. C. Barry, K. Branmann, and M. C. M. Dunne, "Catoptric properties of eyes with misaligned surfaces studied by exact ray tracing," Investigative Ophthalmology \& Visual Science, vol. 38, no. 8, pp. 1476-1484, 1997.

[3] J. Tabernero, A. Benito, V. Nourrit, and P. Artal, "Instrument for measuring the misalignments of ocular surfaces," Optics Express, vol. 14, no. 22, pp. 10945-10956, 2006.

[4] P. Artal and A. Guirao, "Contribution of cornea and lens to the aberrations of the human eye," Optics Letters, vol. 23, pp. 1713-1715, 1998.

[5] P. Artal, A. Guirao, E. Berrio, and D. R. Williams, "Compensation of corneal aberrations by the internal optics in the human eye," Journal of Vision, vol. 1, no. 1, pp. 1-8, 2001.

[6] J. E. Kelly, T. Mihashi, and H. C. Howland, "Compensation of corneal horizontal/vertical astigmatism, lateral coma, and spherical aberration by internal optics of the eye," Journal of Vision, vol. 4, no. 4, pp. 262-271, 2004.

[7] J. Tabernero, A. Benito, E. Alcón, and P. Artal, "Mechanism of compensation of aberrations in the human eye," Journal of the Optical Society of America A, vol. 24, no. 10, pp. 32743283, 2007.

[8] L. Diaz-Santana, C. Torti, I. Munro, P. Gasson, and C. Dainty, "Benefit of higher closed-loop bandwidths in ocular adaptive optics," Optics Express, vol. 11, no. 20, pp. 2597-2605, 2003.

[9] K. M. Hampson, C. Paterson, C. Dainty, and E. A. H. Mallen, "Adaptive optics system for investigation of the effect of the aberration dynamics of the human eye on steady-state accommodation control," Journal of the Optical Society of America A, vol. 23, no. 5, pp. 1082-1088, 2006.

[10] E. J. Fernández and P. Artal, "Dynamic eye model for adaptive optics testing," Applied Optics, vol. 46, no. 28, pp. 6971-6977, 2007.

[11] H. Hofer, P. Artal, B. Singer, J. L. Aragón, and D. R. Williams, "Dynamics of the eye's wave aberration," Journal of the Optical Society of America. A, vol. 18, no. 3, pp. 497-506, 2001.

[12] P. Artal, A. Benito, and J. Tabernero, "The human eye is an example of robust optical design," Journal of Vision, vol. 6, no. 1, pp. 1-7, 2006.

[13] P. Artal and J. Tabernero, “The eye's aplanatic answer," Nature Photonics, vol. 2, no. 10, pp. 586-589, 2008.

[14] J. Porter, A. Guirao, I. G. Cox, and D. R. Williams, "Monochromatic aberrations of the human eye in a large population," Journal of the Optical Society of America. A, vol. 18, no. 8, pp. 1793-1803, 2001.

[15] M. P. Cagigal, V. F. Canales, J. F. Castejón-Mochón, P. M. Prieto, N. López-Gil, and P. Artal, "Statistical description of the wave front aberration in the human eye," Optics Letters, vol. 27, no. 1, pp. 37-39, 2002.

[16] L. N. Thibos, X. Hong, A. Bradley, and X. Cheng, "Statistical variation of aberration structure and image quality in a normal population of healthy eyes," Journal of the Optical Society of America A, vol. 19, no. 12, pp. 2329-2348, 2002. 
[17] J. S. McLellan, P. M. Prieto, S. Marcos, and S. A. Burns, "Effects of interactions among wave aberrations on optical image quality," Vision Research, vol. 46, no. 18, pp. 3009 3016, 2006.

[18] J. F. Castejón-Mochón, N. López-Gil, A. Benito, and P. Artal, "Ocular wave-front statistics in a normal young population," Vision Research, vol. 42, pp. 1611-1617, 2002.

[19] H. W. Babcock, "The possibility of compensating astronomical seeing," Publications of the Astronomical Society of the Pacific, vol. 65, no. 386, p. 229, 1953.

[20] W. Dreher, J. F. Bille, and R. N. Weinreb, "Active optical depth resolution improvement of the laser tomographic scanner," Applied Optics, vol. 28, no. 4, pp. 804-808, 1989.

[21] S. Goelz, J. J. Persoff, G. D. Bittner, J. Liang, C. T. Hsueh, and J. F. Bille, "New wavefront sensor for metrology of spherical surfaces," in Active and Adaptive Optical Systems, vol. 1542 of Proceedings of SPIE, pp. 502-511, 1991.

[22] J. Liang, B. Grimm, S. Goelz, and J. F. Bille, "Objective measurement of wave aberrations of the human eye with the use of a Hartmann-Shack wave-front sensor," Journal of the Optical Society of America A, vol. 11, no. 7, pp. 1949-1957, 1994.

[23] P. M. Prieto, F. Vargas-Martín, S. Goelz, and P. Artal, "Analysis of the performance of the Hartmann-Shack sensor in the human eye," Journal of the Optical Society of America A, vol. 17, no. 8, pp. 1388-1398, 2000.

[24] E. J. Fernández, A. Unterhuber, B. Považay, B. Hermann, P. Artal, and W. Drexler, "Chromatic aberration correction of the human eye for retinal imaging in the near infrared," Optics Express, vol. 14, pp. 6231-6225, 2006.

[25] E. J. Fernández, B. Hermann, B. Považay et al., "Ultrahigh resolution optical coherence tomography and pancorrection for cellular imaging of the living human retina," Optics Express, vol. 16, no. 15, pp. 11083-11094, 2008.

[26] E. J. Fernández and W. Drexler, "Influence of ocular chromatic aberration and pupil size on transverse resolution in ophthalmic adaptive optics optical coherence tomography," Optics Express, vol. 13, no. 20, pp. 8184-8197, 2005.

[27] E. J. Fernández and P. Artal, "Ocular aberrations up to the infrared range: from 632.8 to $1070 \mathrm{~nm}$," Optics Express, vol. 16, no. 26, pp. 21199-21208, 2008.

[28] C. Paterson, I. Munro, and J. C. Dainty, "A low cost adaptive optics system using a membrane mirror," Optics Express, vol. 6, no. 9, pp. 175-185, 2000.

[29] J. Liang, D. R. Williams, and D. T. Miller, "Supernormal vision and high-resolution retinal imaging through adaptive optics," Journal of the Optical Society of America A, vol. 14, no. 11, pp. 2884-2892, 1997.

[30] E. J. Fernández, I. Iglesias, and P. Artal, "Closed-loop adaptive optics in the human eye," Optics Letters, vol. 26, no. 10, pp. 746-748, 2001.

[31] A. Roorda and D. R. Williams, "The arrangement of the three cone classes in the living human eye," Nature, vol. 397, no. 6719, pp. 520-522, 1999.

[32] A. Roorda, F. Romero-Borja, W. J. Donnelly, H. Queener, T. J. Hebert, and M. C. W. Campbell, "Adaptive optics scanning laser ophthalmoscopy," Optics Express, vol. 10, no. 9, pp. 405412, 2002.

[33] B. Hermann, E. J. Fernández, A. Unterhuber et al., "Adaptiveoptics ultrahigh-resolution optical coherence tomography," Optics Letters, vol. 29, no. 18, pp. 2142-2144, 2004.
[34] E. J. Fernández, B. Považay, B. Hermann et al., "Threedimensional adaptive optics ultrahigh-resolution optical coherence tomography using a liquid crystal spatial light modulator," Vision Research, vol. 45, no. 28, pp. 3432-3444, 2005.

[35] E. J. Fernández, A. Unterhuber, P. M. Prieto, B. Hermann, W. Drexler, and P. Artal, "Ocular aberrations as a function of wavelength in the near infrared measured with a femtosecond laser," Optics Express, vol. 13, no. 2, pp. 400-409, 2005.

[36] Y. Zhang, B. Cense, J. Rha et al., "High-speed volumetric imaging of cone photoreceptors with adaptive optics spectral-domain optical coherence tomography," Optics Express, vol. 14, no. 10, pp. 4380-4394, 2006.

[37] R. J. Zawadzki, S. S. Choi, S. M. Jones, S. S. Oliver, and J. S. Werner, "Adaptive optics-optical coherence tomography: optimizing visualization of microscopic retinal structures in three dimensions," Journal of the Optical Society of America A, vol. 24, no. 5, pp. 1373-1383, 2007.

[38] D. Merino, C. Dainty, A. Bradu, and A. G. Podoleanu, "Adaptive optics enhanced simultaneous en-face optical coherence tomography and scanning laser ophthalmoscopy," Optics Express, vol. 14, no. 8, pp. 3345-3353, 2006.

[39] C. E. Bigelow, N. V. Iftimia, R. D. Ferguson, T. E. Ustun, B. Bloom, and D. X. Hammer, "Compact multimodal adaptive-optics spectral-domain optical coherence tomography instrument for retinal imaging," Journal of the Optical Society of America A, vol. 24, no. 5, pp. 1327-1336, 2007.

[40] M. Pircher, R. J. Zawadzki, J. W. Evans, J. S. Werner, and C. K. Hitzenberger, "Simultaneous imaging of human cone mosaic with adaptive optics enhanced scanning laser ophthalmoscopy and high-speed transversal scanning optical coherence tomography," Optics Letters, vol. 33, no. 1, pp. 2224, 2008.

[41] E. J. Fernández, S. Manzanera, P. Piers, and P. Artal, "Adaptive optics visual simulator," Journal of Refractive Surgery, vol. 18, no. 5, pp. S634-S638, 2002.

[42] P. Artal, E. J. Fernández, and S. Manzanera, "Are optical aberrations during accommodation a significant problem for refractive surgery?" Journal of Refractive Surgery, vol. 18, no. 5, pp. S563-S566, 2002.

[43] E. J. Fernández and P. Artal, "Membrane deformable mirror for adaptive optics: performance limits in visual optics," Optics Express, vol. 11, no. 9, pp. 1056-1069, 2003.

[44] P. Artal, L. Chen, E. J. Fernandez, B. Singer, S. Manzanera, and D. Williams, "Neural compensation for the eye's optical aberrations," Journal of Vision, vol. 4, no. 4, pp. 281-287, 2004.

[45] L. Chen, P. Artal, D. Gutierrez, and D. R. Williams, "Neural compensation for the best aberration correction," Journal of Vision, vol. 7, no. 10, pp. 1-9, 2007.

[46] E. A. Rossi, P. Weiser, J. Tarrant, and A. Roorda, "Visual performance in emmetropia and low myopia after correction of high-order aberrations," Journal of Vision, vol. 7, no. 8, article 14, pp. 1-14, 2007.

[47] I. J. Murray, S. L. Elliott, A. Pallikaris, J. S. Werner, S. Choi, and H. J. Tahir, "The oblique effect has an optical component: orientation-specific contrast thresholds after correction of high-order aberrations," Journal of Vision, vol. 10, no. 11, pp. 1-12, 2010.

[48] E. A. Rossi and A. Roorda, "Is visual resolution after adaptive optics correction susceptible to perceptual learning?" Journal of Vision, vol. 10, no. 12, pp. 1-14, 2010. 
[49] L. Sawides, E. Gambra, D. Pascual, C. Dorronsoro, and S. Marcos, "Visual performance with real-life tasks under adaptive-optics ocular aberration correction," Journal of Vision, vol. 10, no. 5, p. 19, 2010.

[50] L. Sawides, S. Marcos, S. Ravikumar, L. Thibos, A. Bradley, and M. Webster, "Adaptation to astigmatic blur," Journal of Vision, vol. 10, no. 12, pp. 1-15, 2010.

[51] R. Sabesan, T. M. Jeong, L. Carvalho, I. G. Cox, D. R. Williams, and G. Yoon, "Vision improvement by correcting higher-order aberrations with customized soft contact lenses in keratoconic eyes," Optics Letters, vol. 32, no. 8, pp. 1000 1003, 2007.

[52] R. Sabesan and G. Yoon, "Visual performance after correcting higher order aberrations in Keratoconic eyes," Journal of Vision, vol. 9, no. 5, article 6, pp. 1-10, 2009.

[53] R. Sabesan and G. Yoon, "Neural compensation for longterm asymmetric optical blur to improve visual performance in keratoconic eyes," Investigative Ophthalmology \& Visual Science, vol. 51, no. 7, pp. 3835-3839, 2010.

[54] L. M. Smithline, "Accommodative response to blur," Journal of the Optical Society of America, vol. 64, no. 11, pp. 15121516, 1974.

[55] K. F. Ciuffreda, "Accommodation and its anomalies," in Vision and Visual Dysfunction, J. R. Cronly-Dillon, Ed., Macmillan, New York, NY, USA, 1991.

[56] L. Stark and Y. Takahashi, "Absence of an odd-error signal mechanism in human accommodation," IEEE Transactions on Biomedical Engineering, vol. 12, no. 3-4, pp. 138-146, 1965.

[57] E. F. Fincham, "The accommodation reflex and its stimulus," British Journal of Ophthalmology, vol. 35, pp. 5-80, 1951.

[58] P. B. Kruger, S. Mathews, K. R. Aggarwala, and N. Sánchez, "Chromatic aberration and ocular focus: fincham revisited," Vision Research, vol. 33, no. 10, pp. 1397-1411, 1993.

[59] K. R. Aggarwala, E. S. Kruger, S. Mathews, and P. B. Kruger, "Spectral bandwidth and ocular accommodation," Journal of the Optical Society of America A, vol. 12, no. 3, pp. 450-455, 1995.

[60] F. W. Campbell and G. Westheimer, "Factors influencing accommodation responses of the human eye," Journal of the Optical Society of America, vol. 49, pp. 568-571, 1959.

[61] W. N. Charman and G. Heron, "Fluctuations in accommodation: a review," Ophthalmic and Physiological Optics, vol. 8, pp. 153-164, 1988.

[62] B. J. Wilson, K. E. Decker, and A. Roorda, "Monochromatic aberrations provide an odd-error cue to focus direction," Journal of the Optical Society of America A, vol. 19, pp. 833839, 2002.

[63] L. Chen, P. B. Kruger, H. Hofer, B. Singer, and D. R. Williams, "Accommodation with higher-order monochromatic aberrations corrected with adaptive optics," Journal of the Optical Society of America A, vol. 23, no. 1, pp. 1-8, 2006.

[64] S. S. Chin, K. M. Hampson, and E. A. H. Mallen, "Role of ocular aberrations in dynamic accommodation control," Clinical and Experimental Optometry, vol. 92, no. 3, pp. 227237, 2009.

[65] S. S. Chin, K. M. Hampson, and E. A. H. Mallen, "Effect of correction of ocular aberration dynamics on the accommodation response to a sinusoidally moving stimulus," Optics Letters, vol. 34, no. 21, pp. 3274-3276, 2009.

[66] E. Gambra, L. Sawides, C. Dorronsoro, and S. Marcos, "Accommodative lag and fluctuations when optical aberrations are manipulated," Journal of Vision, vol. 9, no. 6, article 4, pp. 1-15, 2009.
[67] F. W. Campbell and D. G. Green, "Monocular versus binocular visual acuity," Nature, vol. 208, no. 5006, pp. 191192, 1965.

[68] M. R. Clark and H. D. Crane, "Dynamic interaction in binocular vision," in Eye Movement and the Higher Psychological Functions, J. W. Senders, D. F. Fisher, and R. A. Monty, Eds., pp. 77-88, Erlbaum, New York, NY, USA, 1978.

[69] G. Heron and B. Winn, "Binocular accommodation reaction and response times for normal observers," Ophthalmic and Physiological Optics, vol. 9, no. 2, pp. 176-183, 1989.

[70] G. Heron, B. Winn, J. R. Pugh, and A. S. Eadie, "Twin channel infrared optometer for recording binocular accomodation," Optometry and Vision Science, vol. 66, no. 2, pp. 123-129, 1989.

[71] F. Okuyama, T. Tokoro, and M. Fujieda, "Binocular infrared optometer for measuring accommodation in both eyes simultaneously in natural-viewing conditions," Applied Optics, vol. 32, no. 22, pp. 4147-4154, 1993.

[72] M. Kobayashi, N. Nakazawa, T. Yamaguchi, T. Otaki, Y. Hirohara, and T. Mihashi, "Binocular open-view ShackHartmann wavefront sensor with consecutive measurements of near triad and spherical aberration," Applied Optics, vol. 47, no. 25, pp. 4619-4626, 2008.

[73] S. S. Chin, K. M. Hampson, and E. A. H. Mallen, "Binocular correlation of ocular aberration dynamics," Optics Express, vol. 16, no. 19, pp. 14731-14745, 2008.

[74] E. J. Fernández, P. M. Prieto, and P. Artal, "Wave-aberration control with a liquid crystal on silicon (LCOS) spatial phase," Optics Express, vol. 17, pp. 11013-11025, 2009.

[75] P. M. Prieto, E. J. Fernández, S. Manzanera, and P. Artal, "Adaptive optics with a programmable phase modulator: applications in the human eye," Optics Express, vol. 12, no. 17, pp. 4059-4071, 2004.

[76] P. Howard and B. J. Rogers, Binocular Vision and Stereopsis, vol. 29 of Oxford Psychology Series, Oxford Universit Press, 1995.

[77] R. W. Reading, Binocular Vision: Foundations and Applications, Butterworth-Heinemann, Boston, Mass, USA, 1983.

[78] A. R. Fielder and M. J. Moseley, "Does stereopsis matter in humans?” Eye, vol. 10, no. 2, pp. 233-238, 1996.

[79] R. O'Connor, E. E. Birch, S. Anderson, and H. Draper, “The functional significance of stereopsis," Investigative Ophthalmology \& Visual Science, vol. 51, no. 4, pp. 2019-2023.

[80] J. J. Castro, J. R. Jiménez, E. Hita, and C. Ortiz, "Influence of interocular differences in the Strehl ratio on binocular summation," Ophthalmic and Physiological Optics, vol. 29, no. 3, pp. 370-374, 2009.

[81] J. R. Jiménez, J. J. Castro, R. Jiménez, and E. Hita, "Interocular differences in higher-order aberrations on binocular visual performance," Optometry and Vision Science, vol. 85, no. 3, pp. 174-179, 2008.

[82] J. R. Jiménez, J. J. Castro, E. Hita, and R. G. Anera, "Upper disparity limit after LASIK," Journal of the Optical Society of America. A, vol. 25, no. 6, pp. 1227-1231, 2008.

[83] E. J. Fernández, P. M. Prieto, and P. Artal, "Adaptive optics binocular visual simulator to study stereopsis in the presence of aberrations," Journal of the Optical Society of America A, vol. 27, no. 11, pp. A48-A55, 2010.

[84] B. Julesz, Foundations of Ciclopean Perception, The University of Chicago Press, 1971.

[85] J. V. Lovasik and M. Szymkiw, "Effects of aniseikonia, anisometropia, accommodation, retinal illuminance, and pupil size on stereopsis," Investigative Ophthalmology \& Visual Science, vol. 26, no. 5, pp. 741-750, 1985. 
[86] P. P. Schmidt, "Sensitivity of random dot stereoacuity and Snellen acuity to optical blur," Optometry and Vision Science, vol. 71, no. 7, pp. 466-471, 1994.

[87] C. Schor and T. Heckmann, "Interocular differences in contrast and spatial frequency: effects on stereopsis and fusion," Vision Research, vol. 29, no. 7, pp. 837-847, 1989.

[88] L. K. Cormack, S. B. Stevenson, and D. D. Landers, "Interactions of spatial frequency and unequal monocular contrasts in stereopsis," Perception, vol. 26, no. 9, pp. 1121-1136, 1997.

[89] D. L. Halpern and R. R. Blake, "How contrast affects stereoacuity," Perception, vol. 17, no. 4, pp. 483-495, 1988.

[90] G. Legge and Y. Gu, "Stereopsis and contrast," Vision Research, vol. 29, no. 8, pp. 989-1004, 1989.

[91] C. Wood, "Stereopsis with spatially degraded images," Investigative Ophthalmology \& Visual Science, vol. 3, no. 3, pp. 337-340, 1983.

[92] T. Geib and C. Baumann, "Effect of luminance and contrast on stereoscopic acuity," Graefe's Archive for Clinical and Experimental Ophthalmology, vol. 228, no. 4, pp. 310-315, 1990.

[93] C. Schwarz, P. M. Prieto, E. J. Fernández, and P. Artal, "Binocular adaptive optics vision analyzer with full control over the complex pupil functions," Optics Letters, vol. 36, no. 24, pp. 4779-47781, 2011.

[94] C. Cánovas, P. M. Prieto, S. Manzanera, A. Mira, and P. Artal, "Hybrid adaptive-optics visual simulator," Optics Letters, vol. 35, no. 2, pp. 196-198, 2010.

[95] S. Manzanera, P. M. Prieto, D. B. Ayala, J. M. Lindacher, and P. Artal, "Liquid crystal adaptive optics visual simulator: application to testing and design of ophthalmic optical elements," Optics Express, vol. 15, no. 24, pp. 16177-16188, 2007.

[96] P. A. Piers, E. J. Fenandez, S. Manzanera, S. Norrby, and P. Artal, "Adaptive optics simulation of intraocular lenses with modified spherical aberration," Investigative Ophthalmology \& Visual Science, vol. 45, no. 12, pp. 4601-4610, 2004.

[97] P. A. Piers, S. Manzanera, P. M. Prieto, N. Gorceix, and P. Artal, "Use of adaptive optics to determine the optimal ocular spherical aberration," Journal of Cataract and Refractive Surgery, vol. 33, no. 10, pp. 1721-1726, 2007.

[98] H. Guo, D. A. Atchison, and B. J. Birt, "Changes in throughfocus spatial visual performance with adaptive optics correction of monochromatic aberrations," Vision Research, vol. 48, no. 17, pp. 1804-1811, 2008.

[99] K. M. Rocha, L. Vabre, N. Chateau, and R. R. Krueger, "Expanding depth of focus by modifying higher-order aberrations induced by an adaptive optics visual simulator," Journal of Cataract and Refractive Surgery, vol. 35, no. 11, pp. 1885-1892, 2009.

[100] J. S. Werner, S. L. Elliott, S. S. Choi, and N. Doble, "Spherical aberration yielding optimum visual performance: evaluation of intraocular lenses using adaptive optics simulation," Journal of Cataract and Refractive Surgery, vol. 35, no. 7, pp. 1229-1233, 2009.

[101] R. Legras, Y. Benard, and H. Rouger, “Through-focus visual performance measurements and predictions with multifocal contact lenses," Vision Research, vol. 50, no. 12, pp. 11851193, 2010.

[102] L. Lundström, S. Manzanera, P. M. Prieto et al., "Effect of optical correction and remaining aberrations on peripheral resolution acuity in the human eye," Optics Express, vol. 15, no. 20, pp. 12654-12661, 2007.
[103] D. Williams, G. Y. Yoon, J. Porter, A. Guirao, H. Hofer, and I. Cox, "Visual benefit of correcting higher order aberrations of the eye," Journal of Refractive Surgery, vol. 16, no. 5, pp. S554-S559, 2000.

[104] G. Y. Yoon and D. R. Williams, "Visual performance after correcting the monochromatic and chromatic aberrations of the eye," Journal of the Optical Society of America A, vol. 19, no. 2, pp. 266-275, 2002.

[105] K. M. Rocha, L. Vabre, F. Harms, N. Chateau, and R. R. Krueger, "Effects of Zernike wavefront aberrations on visual acuity measured using electromagnetic adaptive optics technology," Journal of Refractive Surgery, vol. 23, no. 9, pp. 953-959, 2007.

[106] E. Dalimier and C. Dainty, "Use of a customized vision model to analyze the effects of higher-order ocular aberrations and neural filtering on contrast threshold performance," Journal of the Optical Society of America A, vol. 25, no. 8, pp. 20782087, 2008.

[107] E. Dalimier, C. Dainty, and J. L. Barbur, "Effects of higherorder aberrations on contrast acuity as a function of light level," Journal of Modern Optics, vol. 55, no. 4-5, pp. 791-803, 2008.

[108] S. Marcos, L. Sawides, E. Gambra, and C. Dorronsoro, "Influence of adaptive-optics ocular aberration correction on visual acuity at different luminances and contrast polarities," Journal of Vision, vol. 8, no. 13, article 1, 2008.

[109] D. A. Atchison, H. Guo, W. N. Charman, and S. W. Fisher, "Blur limits for defocus, astigmatism and trefoil," Vision Research, vol. 49, no. 19, pp. 2393-2403, 2009.

[110] D. A. Atchison, H. Guo, and S. W. Fisher, "Limits of spherical blur determined with an adaptive optics mirror," Ophthalmic and Physiological Optics, vol. 29, no. 3, pp. 300-311, 2009.

[111] S. L. Elliott, S. S. Choi, N. Doble, J. L. Hardy, J. W. Evans, and J. S. Werner, "Role of high-order aberrations in senescent changes in spatial vision," Journal of Vision, vol. 9, no. 2, pp. $1-16,2009$.

[112] J. Li, Y. Xiong, N. Wang et al., "Effects of spherical aberration on visual acuity at different contrasts," Journal of Cataract \& Refractive Surgery, vol. 35, no. 8, pp. 1389-1395, 2009.

[113] S. Li, Y. Xiong, J. Li et al., "Effects of monochromatic aberration on visual acuity using adaptive optics," Optometry and Vision Science, vol. 86, no. 7, pp. 868-874, 2009.

[114] G. M. Pérez, S. Manzanera, and P. Artal, "Impact of scattering and spherical aberration in contrast sensitivity," Journal of Vision, vol. 9, no. 3, pp. 1-10, 2009.

[115] H. Rouger, Y. Benard, and R. Legras, "Effect of monochromatic induced aberrations on visual performance measured by adaptive optics technology," Journal of Refractive Surgery, vol. 26, no. 8, pp. 578-587, 2010.

[116] P. Artal, S. Manzanera, P. Piers, and H. Weeber, "Visual effect of the combined correction of spherical and longitudinal chromatic aberrations," Optics Express, vol. 18, no. 2, pp. 1637-1648, 2010.

[117] H. Guo and D. A. Atchison, "Subjective blur limits for cylinder," Optometry and Vision Science, vol. 87, no. 8, pp. E549-E559, 2010.

[118] P. Gupta, H. Guo, D. A. Atchison, and A. J. Zele, "Effect of optical aberrations on the color appearance of small defocused lights," Journal of the Optical Society of America A, vol. 27, no. 5, pp. 960-967, 2010.

[119] K. M. Rocha, L. Vabre, N. Chateau, and R. R. Krueger, "Enhanced visual acuity and image perception following 
correction of highly aberrated eyes using an adaptive optics visual simulator," Journal of Refractive Surgery, vol. 26, no. 1, pp. 52-56, 2010.

[120] P. de Gracia, C. Dorronsoro, E. Gambra, G. Marin, M. Hernández, and S. Marcos, "Combining coma with astigmatism can improve retinal image over astigmatism alone," Vision Research, vol. 50, no. 19, pp. 2008-2014, 2010.

[121] H. Hofer, B. Singer, and D. R. Williams, "Different sensations from cones with the same pigment," Journal of Vision, vol. 5, no. 5, pp. 444-454, 2005.

[122] N. M. Putnam, H. Hofer, N. Doble, L. Chen, J. Carroll, and D. R. Williams, "The locus of fixation and the foveal cone mosaic," Journal of Vision, vol. 5, no. 7, pp. 632-639, 2005.

[123] W. Makous, J. Carroll, J. I. Wolfing, J. Lin, N. Christie, and D. R. Williams, "Retinal microscotomas revealed with adaptiveoptics microflashes," Investigative Ophthalmology \& Visual Science, vol. 47, no. 9, pp. 4160-4167, 2006.

[124] A. Raghunandan, J. Frasier, S. Poonja, A. Roorda, and S. B. Stevenson, "Psychophysical measurements of referenced and unreferenced motion processing using high-resolution retinal imaging," Journal of Vision, vol. 8, no. 14, article 14, 2008.

[125] L. C. Sincich, Y. Zhang, P. Tiruveedhula, J. C. Horton, and A. Roorda, "Resolving single cone inputs to visual receptive fields," Nature Neuroscience, vol. 12, no. 8, pp. 967-969, 2009.

[126] E. Dalimier and C. Dainty, "Role of ocular aberrations in photopic spatial summation in the fovea," Optics Letters, vol. 35, no. 4, pp. 589-591, 2010.

[127] K. Y. Li, P. Tiruveedhula, and A. Roorda, "Intersubject variability of foveal cone photoreceptor density in relation to eye length," Investigative Ophthalmology \& Visual Science, vol. 51, no. 12, pp. 6858-6867, 2010.

[128] S. B. Stevenson, A. Roorda, and G. Kumar, "Eye tracking with the adaptive optics scanning laser ophthalmoscope," in Proceedings of the Symposium on Eye-Tracking Research and Applications (ETRA '10), pp. 195-198, ACM, Austin, Tex, USA, March 2010.

[129] S. Poonja, S. Patel, L. Henry, and A. Roorda, "Dynamic visual stimulus presentation in an adaptive optics scanning laser ophthalmoscope," Journal of Refractive Surgery, vol. 21, no. 5, pp. S575-S580, 2005.

[130] Q. Yang, D. W. Arathorn, P. Tiruveedhula, C. R. Vogel, and A. Roorda, "Design of an integrated hardware interface for AOSLO image capture and cone-targeted stimulus delivery," Optics Express, vol. 18, no. 17, pp. 17841-17858, 2010.

[131] A. Roorda, "Adaptive optics for studying visual function: a comprehensive review," Journal of Vision, vol. 11, no. 75, article 6, 2011.

[132] K. M. Hampson, “Adaptive optics and vision," Journal of Modern Optics, vol. 55, no. 21, pp. 3425-3467, 2008. 

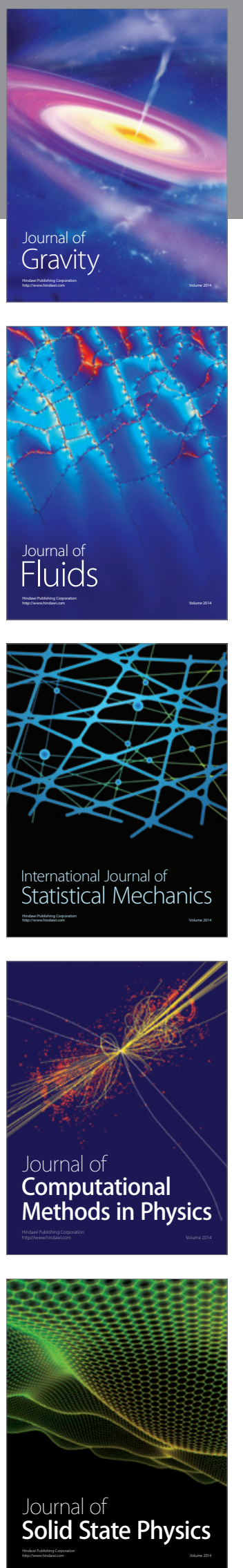
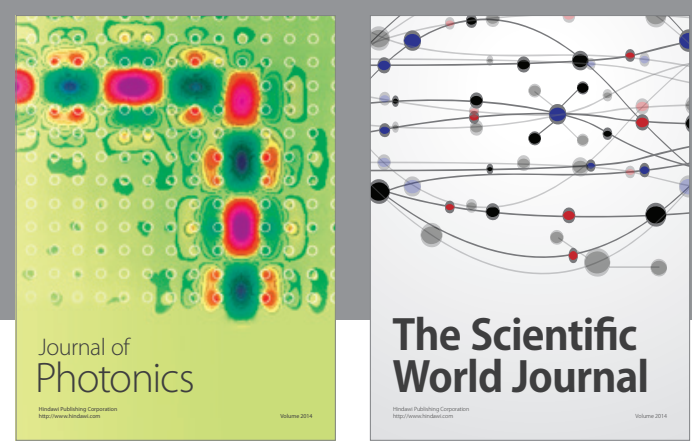

The Scientific World Journal

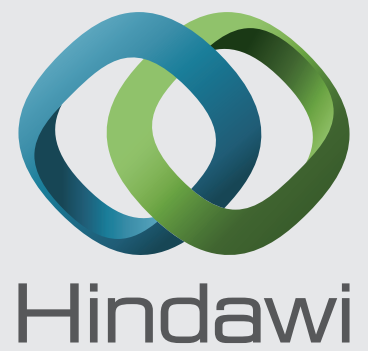

Submit your manuscripts at http://www.hindawi.com
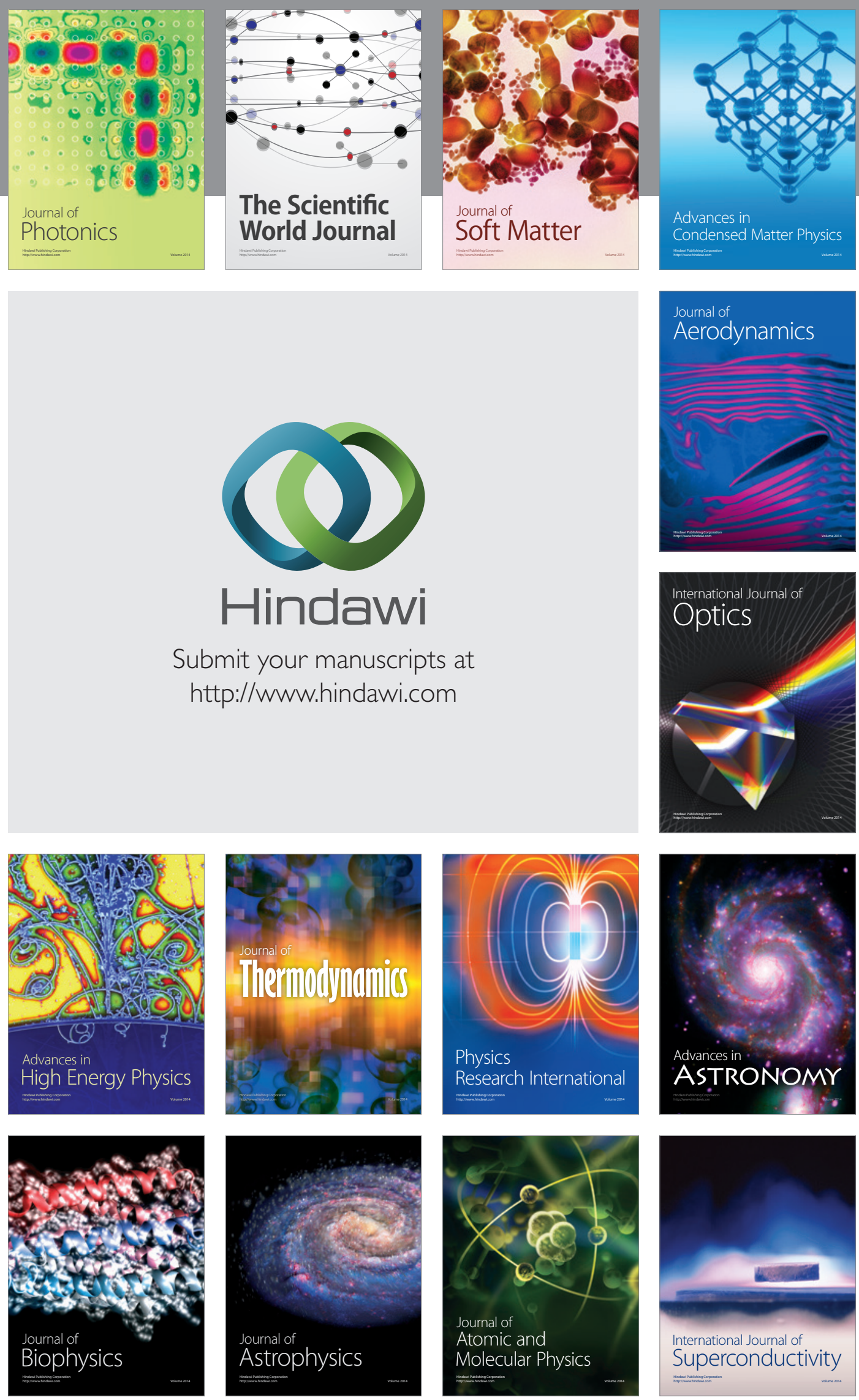
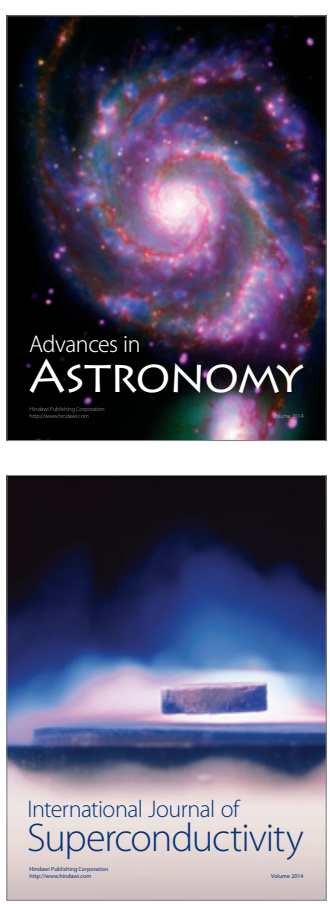